\title{
NUCLEAR RISE OF ROTATION CURVES OF GALAXIES
}

\author{
Y.SOFUE ${ }^{1}$, Y.TUTUI ${ }^{1}$, M.HONMA ${ }^{1}$ AND A.TOMITA ${ }^{2}$ \\ 1. Institute of Astronomy, University of Tokyo, Mitaka, Tokyo \\ 181, Japan (sofue@mtk.ioa.s.u-tokyo.ac.jp) \\ 2.Dept. of Earth and Astron. Sci, Wakayama University, Wakayama \\ 642, Japan
}

\section{CO and $\mathrm{H} \alpha$ Observations}

Rotation curves of spiral galaxies have been thought to rise rigidly in the central a few kpc, while they are flat in the outer regions (Rubin et al 1982; Persic 1996). On the other hand, CO-line and recent $\mathrm{H} \alpha$ observations have revealed steeply rising rotation curves near the nucleus (Sofue 1996,1997; Sofue et al 1997a,b; Rubin et al 1997).

We have obtained high-resolution $\mathrm{CO}$-line position velocity diagrams for the nuclear regions of spiral galaxies, using the Nobeyama $45-\mathrm{m}$ and $\mathrm{mm}$-Array, and also CCD $\mathrm{H} \alpha$ spectroscopy using the Okayama $181-\mathrm{cm}$ telescope. The bulge continuum emission has been carefully subtracted using the pressing method (Fig. 1). Compiling many rotation curves, we investigate their general characteristics in the central regions.

\section{Universal Nuclear Rise of Roation Curves}

Almost all galaxies, among the sample of about 50 galaxies, are found to show a steep rise of rotation velocity within the central 100-300 pc regions

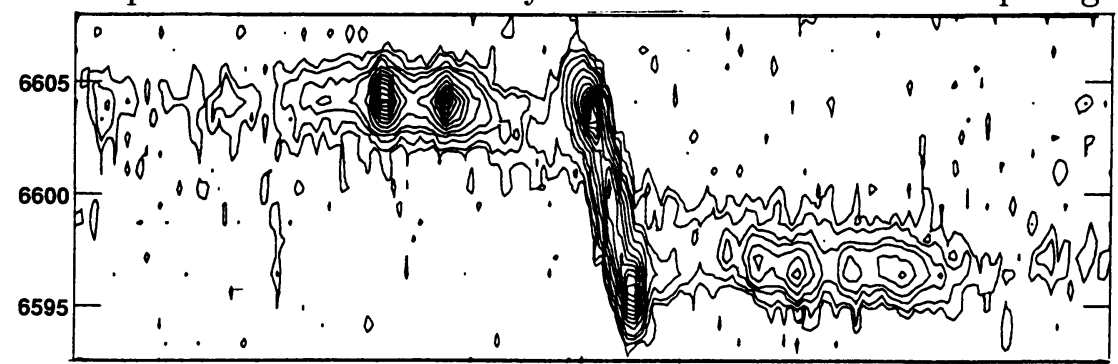

Figure 1. $\mathrm{H} \alpha$ PV diagram for the Sb galaxy NGC 4527. 


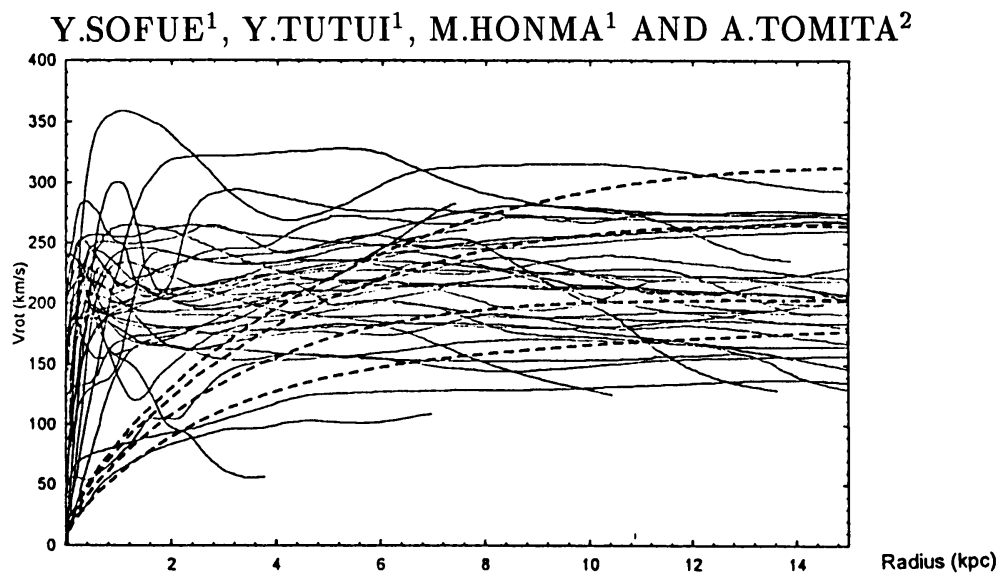

Figure 2. Nuclear-to-outer rotation curves of spiral galaxies. Dashed curves are the so-called 'universal rotation curves' given by Persic et al (1996).

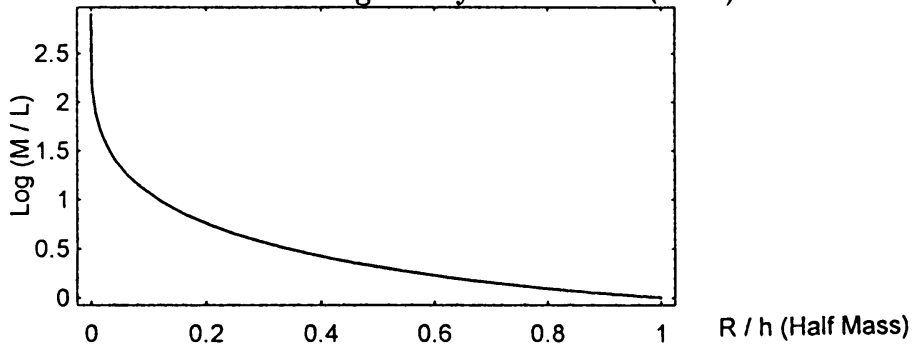

Figure 3. Possible mass-to-light ratio within a bulge, where the surface mass density $\propto e^{-\left(r / r_{e}\right)^{1 / 4}}$ as the rotation curves indicates, and the surface luminosity $\propto e^{-\left(r / r_{b}\right)}$.

(Fig. 2). We stress that the steep nuclear rise will be the general characteristics of rotation curves, universal for disk galaxies.

This indicates that a high-density core of a mass of $\sim 10^{9} M_{\odot}$ and a radius of $\sim 100 \mathrm{pc}$ is present in addition to the usual bulge comcomponent of 0.5 to $1 \mathrm{kpc}$ scale radius. Alternatively, the mass density increases steeply obeying such a law as the $e^{-\left(r / r_{e}\right)^{1 / 4}}$. If the latter is the case, and if the surface luminosity variation is exponential, the mass-to-light ratio increases toward the nuclues, suggesting dark matter around the nucleus (Fig. 3).

\section{References}

Persic, M., Salucci, P., Stel, F. 1996, MNRAS, 281, 27 99, 501.

Rubin, V. C., Ford, W. K., Thonnard, N. 1982, ApJ 261, 439

Rubin V. C., Kenney, J. D. P., and Young, J. S. 1997 AJ 113, 4.

Sofue, Y. 1996, ApJ, 458, 120

Sofue, Y. 1997, PAS J, 49, 17

Sofue, Y., Tomita, A., Honma, M., and Tutui, Y. 1997b, ApJ, submitted.

Sofue, Y., Tutui, Y., Honma, M., Tomita, A., 1997, AJ, 1997a December issue, in press. 